\title{
Editorial: Molecular Pathogenesis of Pneumococcus
}

\author{
Guangchun Bai ${ }^{1 *}$ and Jorge E. Vidal ${ }^{2,3}$ \\ ${ }^{1}$ Department of Immunology and Microbial Disease, Albany Medical College, Albany, NY, United States, ${ }^{2}$ Hubert Department \\ of Global Health, Rollins School of Public Health, Emory University, Atlanta, GA, United States, ${ }^{3}$ Emory Antibiotic Resistance \\ Center, School of Medicine, Emory University, Atlanta, GA, United States
}

Keywords: Streptococcus pneumoniae, pathogenesis, gene regulation, biofilm, co-infection, drug resistance, microbial, macrophages

\section{Editorial on the Research Topic}

\section{Molecular Pathogenesis of Pneumococcus}

Streptococcus pneumoniae has been for decades the number one bacterial killer of children worldwide, but it is also a commensal of the human nasopharynx during childhood. Although vaccination with pneumococcal conjugate vaccines has helped decrease the burden of pneumococcal disease (PD), mortality caused by this pathogen remains high. The introduction of pneumococcal vaccines has also created a niche for vaccine-escape serotypes. Moreover, the rise of multidrug-resistant clones around the world has posed a serious threat in recent years. A better understanding of pneumococcal biology and pathogenesis is needed to further reduce the burden of PD. In the current research topic, nine papers were published for this perspective.

Complex molecules of human epithelia, including the human upper and lower airways, contain $\mathrm{N}$-acetylglucosamine (NAG) that can be metabolized by pneumococcus to survive and persist during colonization, and succeed during pathogenesis. Afzal et al. investigated the transcriptome of the pneumococcal strain D39 growing in media with NAG as a sole source of carbohydrates, and demonstrated upregulated transcription of operons involved in NAG uptake. They confirmed that $n a g A$ and $n a g B$ are essential for the growth of pneumococcus in the presence of NAG and found that NagR regulates genes involved in the metabolism of NAG (Afzal et al.).

Phosphorus and metal ions are essential nutrients for all living organisms (Porcheron et al., 2013). Most bacteria encode a single Pst transporter for the uptake of inorganic phosphate (Pi). However, Zheng et al. demonstrated that $S$. pneumoniae possesses two distinct Pst transporters, Pst1 and Pst2. Interestingly, Pi uptake by these two Pst proteins can be compensated by a $\mathrm{Na}^{+} / \mathrm{Pi}$ co-transporter, NptA, in non-encapsulated S. pneumoniae (NESp) strains. In contrast, either Pst1 or Pst2 is needed for the growth of encapsulated strains. The Pi uptake transporters in S. pneumoniae are tightly controlled by two transcription factors, PhoU1 and PhoU2, to maintain high Pi transport in different Pi availability, which is required for optimal capsule biosynthesis (Zheng et al.).

It is known that AdcR-regulated genes play a role in pneumococcal pathogenesis. Manzoor et al. found that a number of genes belonging to the AdcR and the PsaR regulons are highly upregulated in the presence of $\mathrm{Ni}^{2+}$, suggesting the importance of $\mathrm{Ni}^{2+}$ in pneumococcal virulence. Additionally, most bacteria have developed strategies for iron acquisition from the host hemoglobulin (Hb) or heme (Andrews et al., 2003). S. pneumoniae Spbhp-37 is a cell surface protein and has an high affinity with $\mathrm{Hb}$. The expression of Spbhp-37 is upregulated when S. pneumoniae is grown in media where $\mathrm{Hb}$ is provided as the sole iron source (Romero-Espejel et al.). Therefore, Spbhp-37 might be essential for S. pneumoniae to establish an infection in the host.

Another surface protein of $S$. pneumoniae, pneumococcal surface protein $\mathrm{K}$ (PspK), is known to increase colonization and virulence by NESp during otitis media (OM) episodes 
(Keller et al., 2014). Therefore, PspK is considered as a NESpspecific virulence factor. Interestingly, it was demonstrated that expression of $p s p K$ in an encapsulated pneumococcal strain significantly increased adhesion and invasion of human epithelial cells. Additionally, Murine colonization was increased when a capsule mutant expressed $p s p K$ (Keller et al.). These findings indicate that PspK plays distinct roles in pathogenesis between encapsulated S. pneumoniae and NESp.

It is known that macrophages play an essential role in the recognition and clearance of bacterial infections by either innate immunity or present antigen to establish acquired immunity. Phosphoinositide 3-kinase (PI3K) is an essential regulator for Fc $\gamma$ and CR-mediated phagocytosis (Cox et al., 1999). Protein kinase $\mathrm{B}$ (Akt or $\mathrm{PKB}$ ) is one of the major signal transducers and is a downstream target of PI3K (Alessi et al., 1997). By using immuno-fluorescence microscopy, antibiotic protection assays, and addition of inhibitors of central host cell kinase, new evidence showed that PI3K and Akt play an important role in uptake and killing of $S$. pneumoniae by human THP1 macrophages, which is independent of Fc $\gamma$ or CR-mediated phagocytosis pathways (Kohler et al.).

Through investigating interactions between pneumococcal biofilms and biofilms produced by other human pathogens, Khan et al. discovered that pneumococcal strains produce a factor(s) that efficiently eradicates, i.e., within hours, Staphylococcus aureus biofilms including those produced by methicillin-resistant S. aureus (MRSA) strains. Eradication was more efficient when $S$. pneumoniae and S. aureus had direct contact, which mimicked the condition that the strains would naturally encounter on the human upper airways. Physical-mediated killing did not require production of hydrogen peroxide since a $S$. pneumoniae mutant in $\operatorname{sp} \times B$ gene, encoding for an enzyme involved in production of $\mathrm{H}_{2} \mathrm{O}_{2}$, was able to eradicate $S$. aureus biofilms. Authors hypothesized that research on factors allowing eradication of $S$. aureus will provide alternative therapeutics for $S$. aureus infections (Khan et al.).

Influenza pandemics and seasonal outbreaks have enhanced susceptibility to secondary infection with $S$. pneumoniae.

\section{REFERENCES}

Alessi, D. R., James, S. R., Downes, C. P., Holmes, A. B., Gaffney, P. R., Reese, C. B., et al. (1997). Characterization of a 3-phosphoinositide-dependent protein kinase which phosphorylates and activates protein kinase Balpha. Curr. Biol. 7, 261-269. doi: 10.1016/S0960-9822(06)00122-9

Andrews, S. C., Robinson, A. K., and Rodriguez-Quinones, F. (2003). Bacterial iron homeostasis. FEMS Microbiol. Rev. 27, 215-237. doi: 10.1016/S0168-6445(03)00055-X

Cox, D., Tseng, C. C., Bjekic, G., and Greenberg, S. (1999). A requirement for phosphatidylinositol 3-kinase in pseudopod extension. J. Biol. Chem. 274, 1240-1247. doi: 10.1074/jbc.274.3.1240

Keller, L. E., Friley, J., Dixit, C., Nahm, M. H., and McDaniel, L. S. (2014). Nonencapsulated Streptococcus pneumoniae cause acute otitis media in the chinchilla that is enhanced by pneumococcal surface protein K. Open Forum Infect Dis 1:ofu037. doi: 10.1093/ofid/ofu037
Boianelli et al. developed a computational approach to evaluate the efficacy of treatment with Oseltamivir for flu infection and secondary pneumococcal pneumonia. Their mathematical algorithms revealed that increasing the recommended dose (i.e., $75 \mathrm{mg}$ ) to $150 \mathrm{mg}$, or $300 \mathrm{mg}$, almost doubles the effect of Oseltamivir against secondary bacterial infection. Other aspects of the treatment were also evaluated using their computational approach. Overall, their study warrants clinical trials to determine the efficacy of treatment regimens with Oseltamivir against lethality of secondary pneumococcal infection post influenza (Boianelli et al.).

Macrolides inhibit bacterial protein synthesis by binding to the large 50S ribosomal subunit (Schroeder and Stephens). These antibiotics have been extensively used for treatment of pneumococcal infection, which raises a strong selective pressure contributing to the expansion of macrolide-resistant S. pneumoniae. Macrolide resistance in S. pneumoniae is predominantly due to ribosomal methylation by the gene product encoded by $\operatorname{erm}(B)$ and macrolide efflux by a twocomponent efflux pump encoded by mef (E)/mel (Schroeder and Stephens). Current pneumococcal vaccines are effective in reducing macrolide resistance caused by vaccine serotypes. On the other hand, "serotype replacement" post vaccination might lead to macrolide resistance in new serotypes, which remains a public health concern.

\section{AUTHOR CONTRIBUTIONS}

All authors listed have made a substantial, direct and intellectual contribution to the work, and approved it for publication.

\section{FUNDING}

GB is partly supported by grants of R56AI122763 and R35HL135756 from the National Institutes of Health (NIH). JEV is in part supported by an NIH grant 5R21AI112768-02. The content is solely the responsibility of the authors and does not necessarily represent the official view of the NIH.

Porcheron, G., Garenaux, A., Proulx, J., Sabri, M., and Dozois, C. M. (2013). Iron, copper, zinc, and manganese transport and regulation in pathogenic Enterobacteria: correlations between strains, site of infection and the relative importance of the different metal transport systems for virulence. Front. Cell. Infect. Microbiol. 3:90. doi: 10.3389/fcimb.2013.00090

Conflict of Interest Statement: The authors declare that the research was conducted in the absence of any commercial or financial relationships that could be construed as a potential conflict of interest.

Copyright (C) 2017 Bai and Vidal. This is an open-access article distributed under the terms of the Creative Commons Attribution License (CC BY). The use, distribution or reproduction in other forums is permitted, provided the original author(s) or licensor are credited and that the original publication in this journal is cited, in accordance with accepted academic practice. No use, distribution or reproduction is permitted which does not comply with these terms. 